\title{
PROPIEDADES FISICOQUÍMICAS DE MIELES MONOFLORALES DE ENCENILLO DE LA ZONA ALTOANDINA EN BOYACÁ, COLOMBIA
}

\author{
Guillermo Salamanca Grosso $^{\mathrm{a}, \mathrm{b}, *}$, Mónica Patricia Osorio Tangarife ${ }^{\mathrm{a}}$ y Laura María Reyes Méndez \\ ${ }^{a}$ Grupo de Investigaciones Mellitopalinológicas y Propiedades Fisicoquímicas de Alimentos, Universidad del Tolima, 730006299 \\ Ibagué - TOL, Colombia

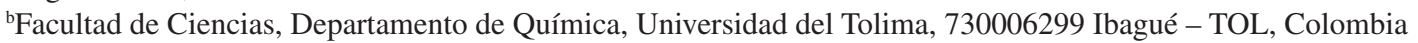

Recebido em 22/10/2016; aceito em 17/04/2017; publicado na web em 11/07/2017

\begin{abstract}
PHYSICOCHEMICAL PROPERTIES OF ENCENILLO MONOFLORAL HONEY FROM HIGHLANDS ANDEAN ZONES IN BOYACÁ, COLOMBIA. Honey is the natural product with sweetening properties more widely known, is produced by bees from the nectar of flowers and secretions from the plants. Its physicochemical properties depend on the season of harvest, environmental factors prevailing in the harvest period and the biogeographic zones. The aim of this work was focused in honeys produced by Weinmannia tomentosa from highlands andean of Colombia, that were characterized on melissopalynology, sensory attributes, composition, physicochemical properties and volatile compounds. Pollen grains at the samples were greater than $45 \%$, conferring a quality of monofloral. Color pfund $69.0 \pm 1.00, \mathrm{pH}$ and total acidity $3.87 \pm 0.04 ; 31.8 \pm 1.40 \mathrm{meq} \mathrm{kg}^{-1}$, still within the standard limits, electrical

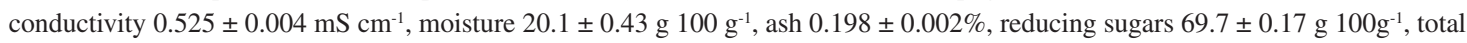
phenols $1570 \pm 14 \mathrm{mg} \mathrm{kg}^{-1} \mathrm{~A}$. Ga, flavonoids $129.4 \pm 1.20 \mathrm{mg} \mathrm{kg}^{-1}$. Tabouret's index $3.75 \pm 0.06$ based on $1.35 \pm 0.03$ relationship fructose/glucose and $0.604 \pm 0.050$ water activity. Honey show a Newtonian's behavior. GC/MS analysis permitted to identify 25 volatile compounds, between alcohols, aldehydes, ketones, esters, hydrocarbons, furans, nitriles, sulfide and terpenes. The study is related as a model for characterization of types of honey and consolidated as the first research on unifloral honey in Colombia.
\end{abstract}

Keywords: Encenillo blossom honey; physicochemical properties; volatile compounds.

\section{INTRODUCCIÓN}

El origen botánico y geográfico de la miel, sigue siendo difícil de determinar, por ello se hace cada vez más necesario estandarizar protocolos de análisis que contribuyan a la verificación de la calidad de éste importante producto de la colmena, determinar su origen floral y reducir el mercado de mieles fraudulentas y suplantadas con jarabes de glucosa que buscan es con sus precios deteriorar el consumo de las mismas. La miel se ha definido como un producto natural con propiedades edulcorantes, elaborada a partir del néctar de las flores o secreciones extraflorales de las plantas vivas que las abejas liban y colectan, transportan, transforman con adición de enzimas especiales para finalmente deshidratar, concentrar y almacenar en los panales..$^{1-5}$ Su maduración, es el resultado complejo de una serie de interacciones al interior de la colmena, que dependen del número de abejas que intervienen el proceso de elaboración, del volumen de néctar almacenado. ${ }^{6}$

La miel es tan diversa como lo es su origen botánico, su naturaleza, composición y sus distintas tonalidades de color; éste parámetro cambia en función de la presencia de aminoácidos libres, antocianinas y antocianidinas, carotenos y xantofilas, flavonas, flavanonas y flavonoles entre otros derivados fenólicos y algunas estructuras propias de alcaloides, que ejercen efectos de atracción entre diversos polinizadores. ${ }^{7,8}$ Debido a su facilidad de digestión, la miel es especialmente valiosa para el consumo directo, su calidad depende de diversos factores, entre los que se debe considerar no solo la flora sino su propia composición. Las propiedades fisicoquímicas y la calidad final están en función de su origen botánico., ${ }^{3,-13}$ Este producto natural, es ampliamente usado en la industria alimentaria, cosmética y farmacéutica. ${ }^{14-16}$

Los atributos de color, aroma, textura y sabor de los alimentos son

*e-mail: salamancagrosso@gmail.com propiedades deseables que marcan la tendencia de los consumidores. Como cualquier producto alimenticio, las mieles poseen propiedades organolépticas que las hacen diferenciables a causa de sus componentes aromáticos dominantes según sea el origen botánico del néctar. El análisis polínico como técnica de diagnóstico y certificación del origen botánico de las mieles si bien es cierto es una herramienta orientativa, pasa a segundo plano en el caso de mieles derivadas de plantas estériles, ${ }^{17}$ además en la evaluación del origen de mieles de mielada, el aporte no es concluyente ${ }^{18}$ por ello el estudio analítico de marcadores químicos ha venido posibilitando el uso de técnicas de análisis multivariado y discriminante de diversos tipos de mieles. ${ }^{19-21}$

El aroma de la miel se atribuye a varios compuestos químicos volátiles $(\mathrm{CV})$ de bajo peso molecular que derivan directamente de las flores visitadas por las abejas. El advenimiento de nuevas técnicas de separación y desarrollo de métodos instrumentales de análisis han permitido identificar más de 150 compuestos., ${ }^{3,19,20,22}$ La diversificación de los productos de la colmena en la actualidad se ha orientado al mercado de los productos diferenciados,, 923 que en el caso de la miel se ha centrado en tipos de mieles florales y monoflorales. La caracterización de mieles especiales da lugar a la implementación de técnicas de análisis melisopalinológico para indagar sobre el origen botánico, ${ }^{11,19,24,25}$ y reconocer sus propiedades fisicoquímicas, nutricionales y terapéuticas en virtud al amplio espectro antibacteriano y actividad como agente inhibitorio de algo más de 80 especies de bacterias. ${ }^{12,26-29}$

En el estudio de CV de mieles, se han identificado más de 600 compuestos de diversa índole, ${ }^{19-20}$ entre aldehídos, alcoholes, ácidos, ésteres, cetonas, hidrocarburos, derivados de furano, pirano, terpenos y sus derivados, además de compuestos cíclicos y derivados de azufre. ${ }^{30-32}$ Los estudios de autenticidad de los tipos de miel, según el origen botánico, han sido orientados a la identificación de compuestos fenólicos como marcadores moleculares (fingerprint). Se conocen aplicaciones en la caracterización de mieles de Eucalipto, Castaño, 
Cítricos, Salvia y Tilo ${ }^{33-34}$ y muestras de Robinia pseudoacacia (Acacia), Ocimum basilicum (Albahaca), Fagopyrum esculentum (Alforfon), Helianthus annuus (Girasol), Tilia cordata (Tilo) y Solidago virgaurea (Vara de oro), ${ }^{33,35}$ cuyos perfiles de componentes volátiles y el uso de técnicas multivariantes en la actualidad han ido permitiendo la generación de patrones tendientes a la identificación de caracteres de diferenciación para el establecimiento del origen

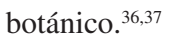

Las mieles adoptan las características organolépticas de las plantas a partir de sus néctares recolectados por las abejas en los nectarios florales y extraflorales, el color, aroma, sabor, densidad, influyen en su composición y propiedades finales. Los componentes de la fracción volátil son relativamente complejos, ya que usualmente se presentan a muy bajas concentraciones y con pobre estabilidad química. ${ }^{5,31,38}$ Entre los productos diferenciados de mayor mercado a nivel mundial, se destacan las mieles de Leptospermum scoparium (Manuka) y Leiospermum racemosum (Kamahi), que han sido usada por los maoríes en Nueva Zelanda desde la antigüedad y que compiten en el mercado de las mieles diferenciadas, como es el caso de las mieles de Robinia pseudoacacia, (Acacia), Gevuina avellana (Avellana), Citrus sp. (Azahar), Eucalyptus sp. (Eucalipto), Lavandula officinalis (Lavanda), Prosopis glandulosa (Mesquite), Corontillo (Escallonia pulverulenta), Quillay (Quillaja saponaria) y Ulmo (Eucryphia cordifolia), entre otros tipos de mieles diferenciadas y sobre las cuales se han generado reportes relacionados con su actividad terapéutica. ${ }^{39,40}$

En Colombia los estudios relacionados sobre calidad de las mieles de Apis mellifera, conforme a su naturaleza, no han sido extensivos, a pesar de ser considerado un país productor. En Colombia, dadas las condiciones de zona de confluencia intertropical, permite la cosecha de mieles en distintos periodos de tiempo dependiendo de las condiciones climáticas locales y los microclimas. Aunque el mercado de mieles florales y monoflorales aún se está posicionado, ${ }^{24,28}$ los estudios sobre calidad y diversidad aún son preliminares. ${ }^{41-45}$ Los tipos de mieles monoflorales de Acacia mangium (Acacio), Smallanthus pyramidalis (Arboloco), Persea americana Mill (Aguacate), Coffea arabica (Café), Ipomoea sp. (Campanita), Citrus sp. (Cítricos), Eucalyptus sp. (Eucalipto), Psidium guajava L. (Guayaba), Inga spectabilis (Guamo), Gliricidia sepium (Matarraton), Mangifera indica (Mango), Trifolium repens L. (Trébol) y Weinmannia sp. (Encenillo), incluyendo las mieles de mielada propias de Quercus humboldtti (Roble), han sido relacionadas a los apicultores y a la cadena de las abejas y la apicultura en Colombia. ${ }^{24,44}$

El Encenillo (Weinmannia tomentosa Don), es una especie arbórea endémica, asociada a bosques de cliserie en los entornos de páramo y subpáramo de Colombia y Venezuela, que crece entre los 2600 a los $3700 \mathrm{msnm} .{ }^{46,47} \mathrm{Su}$ inflorescencia dispuesta en espigas, de tonalidad cremosas a blanca son fuente de néctar, polen y materiales resinosos que Apis mellifera, colectan y benefician para las necesidades de la cría y la protección de la colonia. ${ }^{43,48}$ La especie forestal es de interés apícola debido a los flujos de néctar que permiten cosechar miel monofloral como resultado de la relación planta-insecto. El objetivo de este trabajo, se ha centrado en el estudio de las propiedades fisicoquímicas, viscoelásticas y componentes volátiles de la miel de Encenillo, beneficiada en ecosistemas de bosque de cliserie en zona altoandina de Boyacá.

\section{EXPERIMENTAL}

\section{Muestras}

En el estudio se evaluaron quince muestras de miel centrifugada y de reciente beneficio cosechadas en el periodo agosto-diciembre de 2012 en bosque de cliserie de la zona alto andina de Sotaquirá Boyacá (546`25.5”N; 73¹5`24.8”), en la consociación biogeográfica de bosque húmedo montano bajo ( $b h-M B$ : 2800-3000 msnm; precipitación entre $1000-2000 \mathrm{~mm} / \mathrm{año} ; 12-16^{\circ} \mathrm{C}$ ), y luego de la intensa floración y periodos de flujo de néctar de la especie Encenillo (Weinmannia tomentosa Don).

\section{Melisopalinología}

Este análisis se realizó siguiendo las metodologías y recomendaciones de la comisión internacional de botánica, evitando el proceso de acetólisis. En todos los casos $5 \mathrm{~g}$ de muestra se diluyeron en agua destilada a $45{ }^{\circ} \mathrm{C}$. Los granos de polen se removieron mediante operaciones de centrifugación a $3500 \mathrm{rpm}$ durante $10 \mathrm{~min}$. operando una centrifuga tipo Scientific ${ }^{\circledR}$, el sedimento resultante se resuspendió adicionando $5 \mathrm{~mL}$ de una mezcla de agua y etanol (30:70) y se completó el proceso de separación a $3000 \mathrm{rpm}$ por 5 min. Los pellets del sedimento resultante, se dispusieron en tubos de ensayo, con 2,5 mL de la mezcla agua y glicerina (40:60) por $24 \mathrm{~h}$. centrifugando a $3000 \mathrm{rpm}$ durante $10 \mathrm{~min}$. Los granos se dispusieron en portaobjetos usando la gelatina glicerinada como medio de soporte. La visualización de los granos de polen se realizó haciendo uso de microscopio AmScope ${ }^{\mathrm{TM}}$, con aumento de 40x, con acople a cámara digital tipo Moticam ${ }^{\mathrm{TM}}$, el registro fotográfico se elaboró usando software Motic Image Plus ${ }^{\mathrm{TM}} 2.0 \mathrm{M}$. Se consideró la abundancia relativa porcentual de los granos de polen presentes. . $^{425,26}$

\section{Parámetros fisicoquímicos}

En la caracterización de las muestras se siguieron los protocolos establecidos en el manual de métodos armonizados de análisis de mieles, propuestos por la comisión internacional de la miel, ${ }^{13}$ para los parámetros de humedad, sólidos solubles, $\mathrm{pH}$, acidez, conductividad eléctrica (CE) ${ }^{27}$ densidad óptica, índice de diastasa e hidroximetil furfural. ${ }^{28} \mathrm{El}$ índices de refracción y los sólidos solubles totales, se determinaron en un sistema ATAGO-3T, (Rango 1,3000-1,7100), acoplado al sistema termostático tipo Polyscience-910 (-40 a $100{ }^{\circ} \mathrm{C}$ ), controlado a $20^{\circ} \mathrm{C}$. Los resultados de las observaciones se correlacionaron con los valores correspondientes de sólidos solubles y la humedad. Las determinaciones de actividad de agua, se realizaron en la unidad psicrométrica Decagon $\mathrm{CX}^{\mathrm{TM}}$ (Decagon Devices, Inc., Pullman, USA).

\section{Actividad diastásica}

Las determinaciones de éste parámetro se realizaron haciendo uso del test comercial de Phadebas, ${ }^{13}$ siguiendo los criterios de la casa comercial. Se trabajó sobre $1,0 \mathrm{~g}$ de miel diluida en $100 \mathrm{~mL}$ en disolución tamponada de acetato de sodio $0,1 \mathrm{~mol} \mathrm{~L}^{-1}$ y $\mathrm{pH} 5,2$, con leve calentamiento a $40{ }^{\circ} \mathrm{C}$ y adición de la enzima. Las lecturas se realizaron en la unidad espectrofotométrica UV-Vis Genesys $10 \mathrm{~s}^{\mathrm{TM}}$ (Thermo scientific ${ }^{\mathrm{TM}}$ ), a $620 \mathrm{~nm}$.

\section{Análisis sensorial}

Se realizaron valoraciones relacionadas con los principales atributos de grupos de las muestras de miel, haciendo uso de usando una escala hedónica de clasificación de 0 a 35, conforme a la estrategia planteada por Rtech Laboratorios, (Land O'lakes, St. Paul Minessota), para la National Honey Board (NHB), siguiendo criterios del análisis cuantitativo descriptivo ( $\left.\mathrm{QDA}^{\mathrm{TM}}\right)$. Las muestras fueron evaluadas por 11 jueces entrenados, quienes trabajaron con tres réplicas de miel durante dos sesiones. Cada panelista recibió $10 \mathrm{~g}$ 
de muestra, que evaluó a temperatura ambiente y bajo luz blanca. Entre las características evaluadas se consideró el retrogusto y persistencia floral, dulzor, intensidad del aroma, picor, astringencia, sabor metálico, agentes químicos y ceras.

\section{Color y cromaticidad}

El color (Pfund) se determinó en fotómetro HI 96785 de Hanna Instruments $^{\mathrm{TM}}$. La luminancia (L), croma $(\mathrm{C})$ y cromaticidad rojo/ verde $\left(a^{*}\right)$ y amarillo/azul $\left(b^{*}\right)$ del sistema CIELab, se determinaron a partir de la evaluación de transmitancia entre 400 y 700 nm. en espectrofotómetro UV-Vis Genesys 10s (Thermo scientific ${ }^{\mathrm{TM}}$ ), usando celdas de cuarzo de $10 \mathrm{~mm}$, que se llenaron con muestra evitando la formación de burbujas. Se midió la transmitancia de 10 grupos de longitud de onda, según las determinaciones triestímulo. En las determinaciones se consideró el iluminante C. $2^{\circ}$ (Xn de 98,4, Yn de 100 y $\mathrm{Zn}$ de 118,1).

\section{Densidad óptica}

Las determinaciones se realizaron usando soluciones de miel diluida, en todos los casos $5 \mathrm{~g}$ muestra fueron diluidos en $20 \mathrm{~mL}$ de agua destilada y deionizada y tras la sedimentación del material suspendido mediante centrifugación a $3500 \mathrm{rpm}$ por $5 \mathrm{~min}$. se realizaron mediones de absorbancia a 420 y $635 \mathrm{~nm}$ en el espectrofotómetro UV-Vis Genesys 10s (Thermo scientific ${ }^{\mathrm{TM}}$ ), usando celdas de cuarzo de $10 \mathrm{~mm}$ de paso espectral.

\section{Polarimetría}

Las determinaciones de rotación específica de las muestras objeto de estudio se realizaron luego del proceso de disolución y clarificación, conforme a la metodología descrita por Bianchi ${ }^{16}$ que en resumen corresponde al peso de $10 \mathrm{~g}$ de miel, adición de 2,5 mL de cada uno de los reactivos de Carrez, seguido de la dilución con agua destilada hasta $100 \mathrm{~mL}$. Las mediciones de la desviación de luz polarizada producto de la rotación angular, fueron realizadas usando un polarímetro $Z U Z I^{\mathrm{TM}}$ (Acuatécnica, S.A. Valencia España), con rango de medida $\pm 180^{\circ}$ y divisiones de escala de $0,05^{\circ}$ acoplado a una lámpara monocromática de vapor de sodio a $589,3 \mathrm{~nm}^{28}$

\section{Azúcares}

En las muestras cosechadas se adelantaron determinaciones cuantitativas de los azucares mayoritarios glucosa y fructosa mediante cromatografía líquida de alta eficiencia, (HPLC). El proceso de separación se realizó en un equipo Shimadzu ${ }^{\mathrm{TM}}$ (1C-10AT VP) provisto de detector de índice de refracción (RID-10A). La columna usada fue Rezex ${ }^{\mathrm{TM}}$ RHM monossacharide $\mathrm{H}+(8 \%)$ de $300 \times 7,8 \mathrm{~mm}$. La fase móvil usada fue agua a $0,5 \mathrm{~mL}$ $\min ^{-1}$ presión máxima aplicada 540 psi $\left(40 \mathrm{~kg} \mathrm{f} \mathrm{cm}^{-2}\right)$, el volumen de inyección $20 \mu \mathrm{L}$ en auto-muestreador SIL 10AF (Shimadzu $\left.u^{\mathrm{TM}}\right){ }^{10}$ Se operó disolviendo 2,5 g de cada una de las muestras en $15 \mathrm{~mL}$ de agua (MiliQ), adicionando $5 \mathrm{~mL}$ de metanol (M Tedia-HPLC). La mezcla se homogenizó y completó a volumen en balones aforados de $25 \mathrm{~mL}$. La solución resultante se filtró a través de cartuchos sepack $\mathrm{C}_{18}$ acoplados a filtro de nylon de 4,0 mm y 0,45 $\mu \mathrm{m}$ de poro. Muestras y patrones de fructosa (F) y glucosa $(\mathrm{G})$, se transfirieron a viales de $5 \mathrm{~mL}$ y se almacenaron a $4{ }^{\circ} \mathrm{C}$ hasta el momento del análisis. Patrones individuales de $(\mathrm{F})$ y $(\mathrm{G})$, fueron preparados en el rango 8,0 a $60 \mathrm{mg} \mathrm{mL}^{-1}$ y determinados sus tiempos de retención a 14,5 y $15,9 \mathrm{~min}$. respectivamente.

\section{Índice de cristalización}

Con la finalidad de establecer la tendencia a la cristalización y granulación de la miel se consideraron las determinaciones de glucosa y contenido de humedad, actividad de agua $\left(a_{w}\right)$, además de las relaciones glucosa/humedad, fructosa/glucosa y glucosa-humedad/ fructosa, que fueron incorporados a la expresión asociada al índice de Tabouret (IT) conforme a la expresión que se relaciona, donde (G) y (H) corresponden a la fracción de glucosa y humedad (g/100g), $n$ es un factor adimensional, que depende del contenido de humedad, con valores de 1 si la humedad es superior a $17 \%$ y 2 cuando es inferior.

$$
I T=\frac{G / H}{\left(1-a_{w}\right)^{n}}
$$

\section{Fenoles y flavonoides}

Las determinaciones se realizaron haciendo uso del reactivo de Folin-Ciocalteau (RFC) ${ }^{21,49,50} \mathrm{Se}$ pesaron $5 \mathrm{~g} \pm 0,01 \mathrm{mg}$ de miel que fueron mezclados con metanol y sometidos a extracción continua durante $24 \mathrm{~h}$. A una alícuota de $25 \mu \mathrm{L}$ le fueron adicionados $175 \mu \mathrm{L}$ de agua destilada, $800 \mu \mathrm{L}$ de solución de carbonato de sodio (7,5\%) y $1000 \mu \mathrm{L}$ del reactivo (RFC). La mezcla se dejó reposar por 45 minutos y se leyó la absorbancia a $725 \mathrm{~nm}$. Los resultados se expresan como mg de A.Ga kg-1 de muestra. Los flavonoides, fueron determinados por espectrofotometría usando solución de tricloruro de aluminio y patrones de Quercetina (10 mg del reactivo, que fueron disueltos en etanol al $80 \%$ y posteriormente, diluidos a 10, 20, 40, 80 y $\left.100 \mu \mathrm{g} \mathrm{mL}^{-1}\right) .{ }^{51}$ En las determinaciones $0,5 \mathrm{~mL}$ de cada uno de los extractos de las muestras y de los patrones se mezclaron con 1,5 mL de etanol del 95\%, 0,1 mL de cloruro de aluminio del $10 \%, 0,1 \mathrm{~mL}$ de acetato de potasio $1 \mathrm{~mol} \mathrm{~L}^{-1}$ y $2,8 \mathrm{~mL}$ de agua destilada. La mezcla se incubó a $25^{\circ} \mathrm{C}$ por 30 minutos. Las absorbancias finales se midieron a $415 \mathrm{~nm}$. Los resultados finales se expresaron como equivalentes de quercetina por $\mathrm{mg} \mathrm{kg}^{-1}$ de miel. Las determinaciones se realizaron por espectrofotometría en el equipo UV-Vis Genesys 10s (Thermo scientific ${ }^{\mathrm{TM}}$ ), usando celdas de cuarzo de $10 \mathrm{~mm}$ de paso espectral.

\section{Prolina}

La determinación de éste aminoácido libre procede con la formación de un cromóforo entre la ninhidrina y la prolina en medio ácido. El espectro de absorción del complejo se evaluó en el rango 440 a $560 \mathrm{~nm}$, generando un máximo de absorción a $510 \mathrm{~nm} \cdot{ }^{13}$ Fracciones de $5 \mathrm{~g}$ de miel previamente homogenizada fueron pesadas y disueltas en agua destilada deionizada y transferidos cuantitativamente a balón aforado de $100 \mathrm{~mL}$. Con anterioridad se preparó una curva de calibración usando un patrón acuoso de prolina (40 mg $\left.50 \mathrm{~mL}^{-1}\right)$, que se diluyó a una concentración final de $0,8 \mathrm{mg}_{2} 5 \mathrm{~mL}^{-1}$, usando $1 \mathrm{~mL}$ de patrón diluido en $25 \mathrm{~mL}$ de agua destilada. $0,5 \mathrm{~mL}$ de las muestras y patrones recién preparados se hicieron reaccionar con $1 \mathrm{~mL}$ de ácido fórmico y $1 \mathrm{~mL}$ de solución de ninhidrina (3\% p/p) en monometil éter del etilenglicol.

\section{Propiedades termofísicas}

Los parámetros de conductividad térmica y capacidad calorífica de las muestras estudiadas se midieron en la unidad KD2 Pro (Decagon Devices ${ }^{\mathrm{TM}}$, Pullman WA, USA), haciendo uso de los sensores KS-1 (1,3 mm $\varnothing$ x $60 \mathrm{~mm}$ de longitud) y TR-1 (10 cm x $2,4 \mathrm{~mm} \varnothing)$. La respuesta del equipo fue verificada con el estándar de glicerina de conductividad térmica $0,285\left(\mathrm{~W} \mathrm{~s}^{-1} \mathrm{~K}^{-1}\right)$, con una 
precisión de $\pm 5 \%$ en las mediciones. La capacidad térmica se determinó a través de relaciones entre conductividad térmica y el contenido de humedad. ${ }^{28}$

\section{Calorimetría diferencial}

El comportamiento térmico de las muestras y la estimación de la temperaturas de transición vítrea $(\mathrm{Tg})$, se determinó en un equipo de calorimetría diferencial de barrido (DSC) de TA Instruments, USA, modelo TA 2010, acoplado a un módulo de control térmico Thermal Advantage V. 1.1, disponible a través del software de registro y control Universal Analysis $^{\mathrm{TM}} 2000 \mathrm{~V}$ 4.2E, con accesorios de enfriamiento. La calibración de la unidad se realizó con antelación usando estándar de Indio $\left(\mathrm{Tm} 0156,6{ }^{\circ} \mathrm{C} ; \Delta \mathrm{Hm}=28,71 \mathrm{~J} \mathrm{~g}^{-1}\right)$. En todos los casos, de dispusieron $10 \mathrm{mg}$ de miel, usando viales de aluminio de cierre hermético, que fueron calentaron en el rango $\left(-100\right.$ a $\left.150{ }^{\circ} \mathrm{C}\right)$, con una tasa de barrido de $10{ }^{\circ} \mathrm{C} \mathrm{min}^{-1}$ en atmósfera de nitrógeno. Un vial de aluminio vacío se usó como referencia. En las mediciones se registraron las temperaturas de inicio, el valor medio y final de las transiciones.

\section{Propiedades reológicas}

El comportamiento de las muestras en términos de viscosidad fue evaluado a $25^{\circ} \mathrm{C}$ aplicando un esfuerzo cortante en el rango 0,01 y $300 \mathrm{~s}^{-1}$, en rampas de ascenso y descenso, operando la unidad AR2000 (Advanced Rheometer, TA Instruments ${ }^{\mathrm{TM}}$, New Castle, USA), acoplada a un sistema de análisis de datos (Analysis Software V.5.3.1 de TA Instruments), en una configuración de geometría placa/placa de $40 \mathrm{~mm}$ de diámetro y $700 \mu \mathrm{m}$ de gap. La temperatura del sistema se controló usando una celda tipo Peltier sobre la placa de trabajo. Se evaluaron los resultados tomando como referencia los modelos de viscosidad de Newton y Hershel-Burkley. Adicionalmente, se realizaron determinaciones de flujo dinámico, evaluando el módulo de almacenamiento $\left(\mathrm{G}^{\prime}\right)$, la pérdida de la viscosidad $\left(\mathrm{G}^{\prime \prime}\right)$ y la viscosidad compleja $(\eta *)$ como función de la temperatura a 5,10 y $15^{\circ} \mathrm{C}\left(0,1 \mathrm{~Hz}\right.$ y $10 \mathrm{~s}^{-1}, 700 \mu \mathrm{m}$ gap $)$, a $3 \%$ de deformación en la región viscoelástica lineal. Las relaciones de viscosidad en función de la temperatura fueron determinadas usando la ecuación del modelo de Williams-Landel-Ferry (WLF), fijando la temperatura de referencia en $10{ }^{\circ} \mathrm{C}$, conforme a las relaciones:

$$
a_{T}=\frac{t(T)}{t\left(T_{0}\right)}=\frac{\omega(T)}{\omega\left(T_{O}\right)} \quad \log a_{T}=\frac{-C_{1}\left[T-T_{O}\right]}{C_{2}+\left(T-T_{O}\right)}
$$

Donde es el tiempo requerido para alcanzar la respuesta a una temperatura $\mathrm{T}$ y el tiempo necesario para alcanzar la respuesta a la temperatura de referencia, es la frecuencia, $\mathrm{C} 1$ y $\mathrm{C} 2$ son constantes dependientes de la temperatura, conforme a las directrices de la literatura y el principio de superposición de las relaciones tiempo temperatura (TTS). ${ }^{49,52}$

\section{Componentes volátiles}

Las muestras de miel (10 g) previamente homogenizadas y filtradas, les fue adicionado $200 \mu \mathrm{L}$ de 2 -pentanol a una concentración final de $10 \mu \mathrm{L} \mathrm{mL}^{-1}$ como estándar interno. A continuación fueron dispuestas en un frasco de purga, adaptado a baño termostático a $45^{\circ} \mathrm{C}$. A través de tubo de vidrio con terminal porosa se hizo burbujear y circular nitrógeno puro a $100 \mathrm{~mL} \mathrm{~min}{ }^{-1}$ para volatilizar los compuestos volátiles, que fueron retenidos en un polímero poroso (100 mg) tipo Tenax TA, de 20 - 35 mesh, dispuesto en un tubo al final del sistema. ${ }^{53,54}$

\section{Desorción de volátiles}

Las fracciones volátiles fueron desorbidas en la unidad de desorción térmica (TD Turbo-Matrix, Perkin Elmer ${ }^{\mathrm{TM}}$ ), usando helio como gas de arrastre, con flujo de $10 \mathrm{~mL}$ a $220{ }^{\circ} \mathrm{C}$ por 16 minutos; la fracción volátil entonces se crioconcentró a $-30{ }^{\circ} \mathrm{C}$ y posteriormente fue transferida a la cabeza de una columna capilar (DB-WAX, de SGE, Melbourne, Australia, $60 \mathrm{~m}$ de longitud, 0,32 mm d.i., 1,0 $\mu \mathrm{m}$ de grosor en el film), en un cromatógrafo de gases (GC), con rampa de calentamiento de $99{ }^{\circ} \mathrm{C} \mathrm{s}^{-1}$ hasta $250{ }^{\circ} \mathrm{C}$. La unidad de espectrometría de masas Finnigan Trace mass spectrometer (Thermo Quest, Austin, TX, USA), que fue usada en el análisis. Se usó helio a $1 \mathrm{~mL} \mathrm{~min}^{-1}$ como gas de acarreo. El proceso de separación se completó trabajando a temperatura programada con incremento desde $40{ }^{\circ} \mathrm{C}$ (tiempo sostenido $2 \mathrm{~min}$ ) a $190{ }^{\circ} \mathrm{C} \mathrm{a} 4{ }^{\circ} \mathrm{C} \mathrm{min}{ }^{-1}(11 \mathrm{~min}$. sostenido) y finalmente a $220^{\circ} \mathrm{C} \mathrm{a} 8{ }^{\circ} \mathrm{C} \mathrm{min}^{-1}$ ( $8 \mathrm{~min}$. sostenido). La interface del espectrómetro de masas y fuente térmica en su orden se mantuvieron a 250 y $200{ }^{\circ} \mathrm{C}$. Los espectros de masas por impacto electrónico fueron registrados en modo ionización a $70 \mathrm{eV}$ en un rango $\mathrm{m} / \mathrm{z}$ de $33-433$. Las determinaciones se realizaron por triplicado.

La identificación de los compuestos separados se realizó por comparación directa entre los espectros de masas de cada uno de los compuestos aislados y la de estándares puros, los tiempos de retención y los índices lineales de retención de Kovats de la serie de alcanos lineales (Fluka Buchs, Schwiez, Switzerland, $n-\mathrm{C}_{8}$ a $n-\mathrm{C}_{20}$ ), a partir de datos reportados en la literatura. ${ }^{5,38,55}$ Se calcularon los Índice de Kovats de cada compuesto (índices de retención relativos) y se compararon con datos de la literatura y con los tiempos de retención de compuestos volátiles obtenidos de las muestras con los de patrones puros de (2-metil-1-propanol, 2-metil-1-butanol + 3-metil-1-butanol, benzaldehído, heptano, octano, 3-hidroxi-2-butanona, furfural, dimetil sulfuro y $D$-limoneno).

\section{Análisis estadístico}

Los datos fueron analizados haciendo uso de paquetes estadísticos Statistica $^{\mathrm{TM}}$ (Versión 10.0), siguiendo criterios de análisis de varianza de una vía (ANOVA), con un nivel de significancia del $95 \%$ de confianza, en virtud al número de muestras analizadas y las réplicas de las determinaciones, para analizar diferencias significativas entre muestras de miel. Los ensayos se realizaron por triplicado, los resultados finales de las determinaciones de cada uno de los parámetros se expresan como su promedio y desviación estándar.

\section{RESULTADOS Y DISCUSIÓN}

El Encenillo (Weinmannia tomentosa Don), es una especie arbórea pequeña de $8 \mathrm{~m}$ de alto y $20 \mathrm{~cm}$ de diámetro, sus hojas son imparipinnadas y opuestas, de borde aserrado, con estípulas connadas de la familia Cunoniaceae. Inflorescencias en racimos densos y angostos que semejan espigas axilares, de 4 a $10 \mathrm{~cm}$ de largo, ejes pubescentes. Flores pequeñas, glabras y blanquecinas, el pedicelo es de 1 a $2 \mathrm{~mm}$; el cáliz presenta de 4 a 5 sépalos triangulares, unidos en la base; corola con 4 pétalos, oblongos, de 1 a $2 \mathrm{~mm}$; con numerosos estambres que sobresalen, tal como se evidencia (Figura 1A-B). La importancia apícola de la especie obedece al aporte de polen, néctar y exudaciones, que las abejas colectan para la elaboración de propóleos. Florece en los meses de Agosto a Noviembre. El polen de las mieles representan frecuencias dominantes superiores al $75 \%$, los recuentos han sido superiores a 120.000 granos de polen/10 gramos de miel. En el sistema instalado en la zona de estudio, la miel se beneficia de colonias tipo Langstroth, con rendimientos de 25 a $30 \mathrm{~kg} /$ colmena. 
El análisis melisopalinológico de los tipos de mieles monoflorales en general se presenta como garantía de su origen botánico. El estudio microscópico del sedimento resultante del proceso de centrifugación reveló la presencia de granos pequeños de 10 a $25 \mu \mathrm{m}$, granos simétricos, trizonocolporados y tectados, finamente recticulados y estriados, tamaño global $12,8 \pm 0,10 \mu \mathrm{m}$, el grosor de la exina $1,30 \pm 0,01 \mu \mathrm{m}$. (Figura 1C). El polen observado, es coincidente con las preparaciones polínicas y atlas de referencia de Weinmannia tomentosa Don. (Preparación CP-UT-55/Universidad del Tolima).

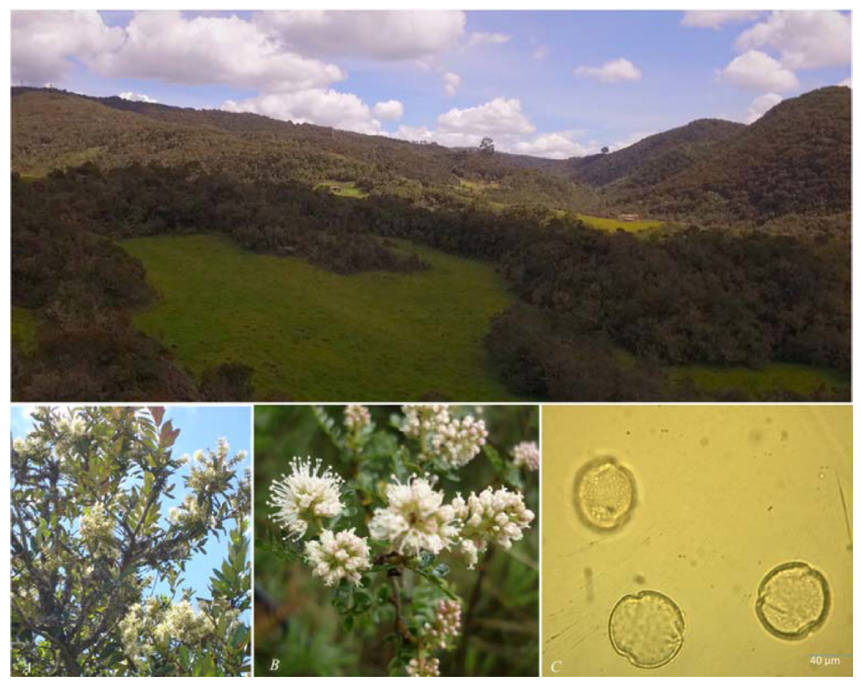

Figura 1. Características de entorno zona altoandina de Boyacá bosque de Encenillo. (A) Aspecto morfológico de la planta, (B) inflorescencia y (C) polen

\section{Atributos sensoriales}

Estas mieles son de tonalidad ámbar claro, en términos palatables, se acompañan de un marcado acento boscoso y tenue fragancia floral que es persistente aún en anaquel, con sensación suave y dulce delicado y tonos ácidos débiles persistentes hasta después de su consumo, de sensación canforosa que perdura en el retrogusto. Aroma característico de las mieles altoandinas con notas agradables a flores de campo con tonos resinosos muy ligeros. Ausencia de sabores metálicos, reducido sabor a cera de panal y leves notas fenólicas, picor característico de mieles recién beneficiadas. En la Figura 2, se ilustra el perfil sensorial, observado en éste tipo de miel monofloral.

\section{Propiedades generales}

Las mieles evaluadas son de color ámbar claro, este tipo de muestras puede variar de 68.0 a 70.0 el valor medio de $69.0 \pm 1.00$, se correlaciona con los tipos de mieles ámbar claro (LA). Los parámetros cromáticos CIELab, no son frecuentes en los estudios de calidad de mieles, en éste caso se han incluido como información complementaria, la luminancia media de $59.6 \pm 1.48$, la cromaticidad rojo/verde $\left(a^{*} 16.5\right.$ a 18.4$)$, amarillo/azul $\left(b^{*} 79.8 \pm 1.90\right)$ y el croma (C $81.7 \pm 2.10)$. Las muestras estudiadas, como ocurre con algunos tipos de miel, son de naturaleza compleja con saturación de azúcares que confieren condiciones metaestables, susceptible de cristalización espontánea y que trae en algunos casos la formación de fases. La fracción de azúcares representan niveles hasta del 95 $\%$ de los sólidos totales y le otorgan propiedades físicas singulares como el índice de refracción, actividad de agua $\left(a_{w}\right)$, capacidad de hidratación, viscosidad, poder rotatorio, conductividad y color así como la tendencia a la granulación. Se ha demostrado que $\left(a_{w}\right)$ es marcadamente dependiente del contenido de glucosa y fructosa. ${ }^{56} \mathrm{La}$

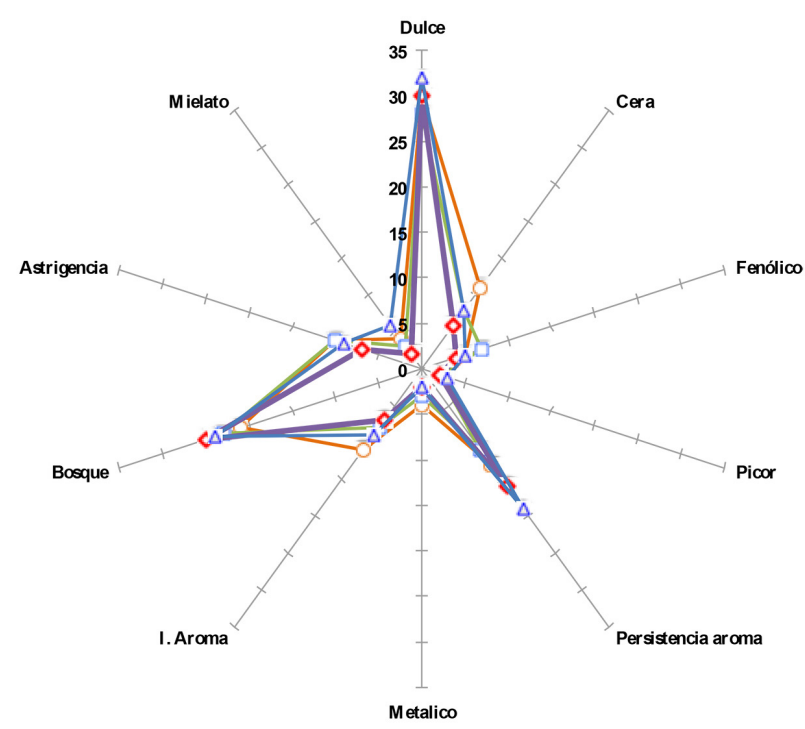

Figura 2. Perfil sensorial relativo a los principales atributos de mieles de Encenillo de la zona altoandina de Boyacá

fracción media para los sólidos solubles de las mieles evaluadas fue

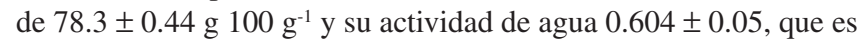
propia de los alimentos de humedad intermedia. La conductividad eléctrica (C.E: $\mathrm{mS} \mathrm{cm}^{-1}$ ), que es una propiedad intrínseca está relacionada con el contenido de cenizas. Los parámetros evaluados son comparables con otros tipos de mieles y dan cuenta del grado de madurez alcanzado por el producto tras el proceso de operculación de la miel en los panales. . $, 23,31,42-44,57-59^{-1}$

Los sólidos iónicos conductores totales (TCD), oscilaron en promedio entre $0.261 \pm 0.003 \mathrm{ppm}$, concordante con la fracción de cenizas y la conductividad eléctrica de las mieles, con $0.525 \pm 0.004$

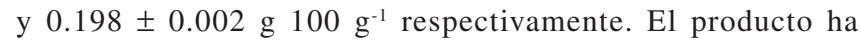
exhibido propiedades ácidas, con un $\mathrm{pH} 3.87 \pm 0.04 \mathrm{y}$ acidez total $31.8 \pm 1.41$ meq kg-1; la rotación óptica media de $-20.8 \pm 0.63$, los azúcares mayoritarios Fructosa (F) y Glucosa $(\mathrm{G})$ con $40.1 \pm 0.30$ y

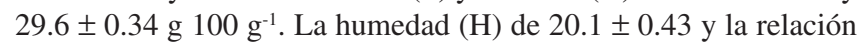
(F/G) $1.35 \pm 0.03$ y $(\mathrm{G} / \mathrm{H})$ de $1.30 \pm 0.03$. Los parámetros indicadores de frescura de la miel, tradicionalmente se han centrado en el contenido de hidroximetilfurfural (HMF) e índice de diastasa (ID), principalmente. La presencia de diastasas ( $\alpha$-amilasa, amilo clástica y $\beta$-amilasa, sacarogénica), influencian el contenido final de azúcares

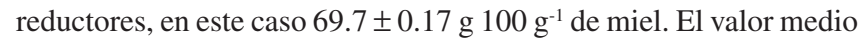
de ID es del orden de $11.8 \pm 0.30$ y el HMF medio es del orden de $44.7 \mathrm{mg} \mathrm{kg}^{-1}$. Algunos de los parámetros evaluados son coincidentes con los de mieles de Leptospermum scoparium, (Manuka), ${ }^{9}$ Eucryphia cordifolia (Ulmo), Quillaja saponaria (Quillay) y Encelia canescens (Corontillo). ${ }^{31}$

Los estudios relacionados con la actividad antioxidante y capacidad para inhibición de radicales libre en mieles, ha sido relacionada con el contenido de fenoles y flavonoides, ${ }^{60}$ éstos componentes son incorporados principalmente desde el néctar y el polen. El contenido medio de flavonoides ( $\mathrm{mg} \mathrm{kg}^{-1}$ Quercetina) y fenoles totales ( $\mathrm{mg} \mathrm{kg}^{-1} \mathrm{~A}$. Gálico), en las muestras evaluadas oscilaron entre $129.4 \pm 1.20$ y $1570 \pm 1.4$ (Tabla 1 ). Estos valores son comparables con los hallados en mieles portuguesas y africanas, con 226.2 a 727.8 y 52.2 a 789.6 ( $\mathrm{mg} \mathrm{kg}^{-1}$ A. Gálico), ${ }^{50}$ aunque se conocen otros estudios donde el parámetro oscila entre 102.1 a $1085 \mathrm{mg} \mathrm{kg}^{-1}{ }^{61}$ El tenor de fenoles de la miel de Encenillo es indicador de una importante actividad antioxidante total (AAT).

La prolina ha sido sugerida como indicador de calidad de la miel, es el aminoácido libre con mayor representación respecto de 
otros aminoácidos, permite identificar tipos de mieles adulteradas y al esclarecimiento del origen botánico, además de relacionarse con el grado de madurez. Éste aminoácido proviene principalmente de la actividad metabólica de las abejas y del polen de las plantas que pecorean. ${ }^{62}$ El Codex alimentarius, ha sugerido un valor guía de $180 \mathrm{mg} \mathrm{kg}^{-1}$ y en las mieles de Encenillo el valor medio observado es de $368 \pm 37.2 \mathrm{mg} \mathrm{kg}^{-1}$, que es mayor respecto de valores medios observados en otros tipos de mieles. ${ }^{63-65}$

\section{Propiedades ópticas}

La miel como en otros productos de origen natural, tiene la propiedad de hacer girar la luz en un plano de polarización. Esta propiedad depende en gran medida de los tipos y proporciones de azúcares mayoritarios presentes. Las mieles florales en general son levógiras, efecto derivado de la actividad óptica de la fructosa, (rotación específica -92.4) a $25^{\circ} \mathrm{C} .{ }^{5} \mathrm{La}$ actividad óptica de las mieles de Encenillo observadas en éste trabajo, estuvieron en el rango (-21.0 a -20.6$)$ con un promedio de $(-20.8 \pm 0.16)$; estos resultados son consistentes con el perfil de monosacáridos encontrado en trabajos relacionados con mieles multiflorales que mostraron un poder rotatorio del orden de $-14.8 \pm 4.90,{ }^{66}$ Medicago sativa (Alfalfa) $-18.1 \pm 2.70,{ }^{11}$ y Robinia pseudoacacia (Robinia) $-16.0,{ }^{67}$ Eucalyptus sp. (Eucalipto) $-13.4 \pm 2.30,{ }^{25}$ Taraxacum campylodes (Diente león) $-10.0 \pm 2.40 .^{68}$

\section{Índices de cristalización}

El proceso de cristalización observado en algunos tipos de miel no un fenómeno natural, dependiente de su composición, de la humedad y de la relación entre los azucares reductores, principalmente glucosa y fructosa. En este proceso intervienen otros factores como el tipo de envase y las condiciones térmicas de entorno, que dan paso a cambios de fase en el producto, que se relacionan con la tendencia a nucleación de los azucares y generan transiciones de primer orden con generación de estructuras no necesariamente cristalinas, pero si amorfas pudiendo alcanzar el estado vítreo o gomoso, donde la movilidad molecular de los componentes cambia drásticamente y se alteran las propiedades mecánicas. ${ }^{69}$

La relación fructosa/glucosa, ha sido descrita como índice de cristalización; usualmente ésta relación oscila entre 1.32 y 1.38 . El valor medio es la miel evaluada fue de $1.35 \pm 0.03$. Las mieles cuya relación fructosa/glucosa sean superiores a 2.0 son indiferentes al proceso de cristalización. El índice de Jacson/Silsbee, relacionado con el contenido de glucosa, humedad y fructosa (G-H/F), en las mieles propias del trabajo fueron del orden de 0.190 . El valor medio del índice Tabouret es de 3.27 (Tabla 1). En principio las mieles recién cosechadas son líquidas, con una marcada tendencia a la granulación, debido principalmente a la presencia de granos de polen que facilitan su nucleación, como ocurre en la mayoría de las mieles. ${ }^{59,70}$

\section{Propiedades termofísicas}

La miel como la mayoría de los alimentos conducen el calor de manera deficiente, los procesos de transmisión por efectos de conducción son lentos, la difusividad $(\alpha)$, capacidad calorífica $(\mathrm{Cp})$ y conductividad térmica $(\mathrm{k})$ están influenciadas por el contenido de humedad, presencia o ausencia de dextrinas, sólidos solubles totales y fracción nitrogenada principalmente. ${ }^{55}$ En las muestras objeto de estudio los valores medios para $(\mathrm{Cp})$ y $(\alpha)$ son $2.10 \pm 0.05 \mathrm{~kJ} \mathrm{~kg}^{-1}{ }^{\circ} \mathrm{C}^{-1}$ y $4.51 \times 10^{-5} \mathrm{~m} \mathrm{~s}^{-1}$ para una densidad de $1.4129 \mathrm{~g} \mathrm{~mL}^{-1}$ que están en el rango de valores reportados en mieles colombianas de Boyacá y Tolima, entre 1.93 y $2.01\left(\mathrm{Cp}: \mathrm{kJ} \mathrm{kg}^{-1} \mathrm{~K}^{-1}\right)$ y $0.349 \pm 0.007$ $\left(\mathrm{k}: \mathrm{W} \mathrm{m}^{-1} \mathrm{~K}^{-1}\right)$. Entre tanto los termogramas de calorimetría diferencial de las mieles de Encenillo (Figura 3), muestran dos endotermos bien definidos, el primero inicia en $-50.4 \pm 0.22{ }^{\circ} \mathrm{C}$, valor medio $(-45.3 \pm 0.10)$ y finaliza $-42.0 \pm 0.18^{\circ} \mathrm{C}$.

En los productos azucarados como en mieles, en la condición de equilibrio termodinámico por debajo de la licuefacción, se presenta el fenómeno de cristalización del agua y la fracción de azucares, en un proceso que converge a la transición vítrea $(T g)$ parámetro físico que en la actualidad se ha recomendado en estudios de detección de la suplantación y adulteración de mieles. ${ }^{71}$ Los valores medios de $T g$ observados en la miel de Encenillo, son significativamente mayores en comparación a los valores reportados en muestras de miel griega $(-34.6$ y -47.2$){ }^{\circ} \mathrm{C},{ }^{56}$ australianas $(-40 \mathrm{y}-46){ }^{\circ} \mathrm{C},{ }^{72} \mathrm{e}$ indias $(-33.6 \mathrm{y}$ -51.1) ${ }^{\circ} \mathrm{C} .{ }^{73} \mathrm{La}$ variabilidad de $T g$ esta en función del contenido de humedad y de la fracción de azucares. ${ }^{74}$

\section{Propiedades reológicas}

Las propiedades relógicas de la miel están relacionadas con su naturaleza y composición, los efectos de pseudoplasticidad de este tipo de productos, es propio de la adición de melazas y otros edulcorantes como agentes de suplantación y adulteración. El efecto tixotrópico de algunas mieles, se relacionan con la presencia de algunos tipos de proteínas en la matriz, entre tanto la presencia de dextrinas son las causantes de efectos dilatantes. En el estudio la viscosidad media de las mieles evaluadas, han sido de $(5.08 \pm 0.09)$ Pa.s a $25^{\circ} \mathrm{C}$, con una tixotropía de $11410 \pm 671 \mathrm{~Pa} \mathrm{~s}^{-1}$. El comportamiento en general de las muestras se ajustan al modelo de un fluido newtoniano, propio de soluciones concentradas cuyos componentes son de bajo peso molecular, donde se presenta un aumento de la viscosidad al reducir las condiciones térmicas del fluido a diferentes velocidades de cizallamiento debido a la reducción de las fuerzas hidrodinámicas y pérdida de la fricción molecular. ${ }^{10,72}$ La naturaleza compleja de la miel, en virtud al número de componentes que la constituyen y el estado termodinámico de no equilibrio de la misma, propicia cambios permanentes que son dependientes del tiempo. ${ }^{75}$

Las propiedades viscoelásticas de la miel de Encenillo, puede ser descrita en términos de los módulos de almacenamiento $\left(\mathrm{G}^{\prime}\right)$ y pérdida de la viscosidad $\left(\mathrm{G}^{\prime \prime}\right)$ a 5,10 y $15^{\circ} \mathrm{C}$. En éstas condiciones los valores asociados a éstos parámetros presentan un incremento en la medida en que se incrementa la frecuencia del proceso oscilatorio. En la Figura 4, se muestra la relación entre la frecuencia y los módulos mecánicos. Los parámetros $\left(\mathrm{G}^{\prime \prime}\right)$ y $\left(\mathrm{G}^{\prime}\right)$ son de la forma $\left[G^{\prime}=K^{\prime}(\omega)^{n}\right]$ y $\left[G^{\prime \prime}=K^{\prime \prime}(\omega)^{n^{\prime \prime}}\right]$. $\mathrm{K}^{\prime}, n^{\prime}$ y $\mathrm{K}^{\prime \prime}, n^{\prime \prime}$, representan la pendiente media de la relación entre la frecuencia $(\omega)$, los módulos de almacenamiento y la pérdida de la viscosidad; $n^{\prime}$ y $n^{\prime \prime}$ son los exponentes de cada una de las relaciones indicadas. El análisis dinámico a las temperaturas son coincidentes con el marcado comportamiento newtoniano de las muestras evaluadas (Tabla 2). En el rango 0.1 a $10 \mathrm{~Hz} \mathrm{~s}^{-1}$ la viscosidad compleja $\left(\eta^{*}\right)$, se mantiene independiente de la frecuencia aplicada, con $\left(\mathrm{G}^{\prime \prime}\right)>>\left(\mathrm{G}^{\prime}\right)$, condición propia de líquidos newtonianos. ${ }^{49,52}$ Se conocen algunos reportes de mieles que se apartan del comportamiento newtoniano, como ocurre en algunos tipos de mieles lituanas y las monoflorales de Calluna vulgaris, los resultados de las determinaciones de las mieles de Encenillo, son consistentes con las propiedades de viscosidad de muestras de diferentes orígenes botánicos que se describen como fluidos newtonianos. ${ }^{29,56,69}$

En la valoración de la viscosidad de fluidos que se ajustan las relaciones de ley de potencia, como es el caso de algunos tipos de miel, la relación de Williams-Landel-Ferry (WLF), resulta de particular interés cuando se considera el principio de superposición de tiempo temperatura (TTS). ${ }^{49,52,56}$ Los resultados de los ensayos reológicos a una temperatura pueden superponerse con otros obtenidos en 
Tabla 1. Parámetros fisicoquímicos asociados a la calidad de mieles de Encenillo de la zona altoandina de Boyacá

\begin{tabular}{|c|c|c|c|c|}
\hline Parámetros & Unidades & Mínimo & Máximo & Promedio $\pm \mathrm{ds}$ \\
\hline I. Refracción & - & 1.4844 & 1.4877 & $1.4864 \pm 0.0010$ \\
\hline${ }^{\circ}$ Brix & g $100 \mathrm{~g}^{-1}$ & 77.4 & 78.8 & $78.3 \pm 0.44$ \\
\hline Densidad & $\mathrm{g} \mathrm{mL}^{-1}$ & 1.4099 & 1.4160 & $1.4129 \pm 0.0020$ \\
\hline R. Específica & & -21.0 & -20.6 & $-20.8 \pm 0.16$ \\
\hline C.E & $\mathrm{mS} \mathrm{cm} \mathrm{cm}^{-1}$ & 0.517 & 0.531 & $0.525 \pm 0.004$ \\
\hline Cenizas & g $100 \mathrm{~g}^{-1}$ & 0.194 & 0.201 & $0.198 \pm 0.002$ \\
\hline $\mathrm{TCD}$ & ppmil & 0.263 & 0.271 & $0.261 \pm 0.003$ \\
\hline C. Térmica & 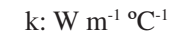 & 0.339 & 0.356 & $0.349 \pm 0,007$ \\
\hline Capacidad calorífica & $C p: \mathrm{kJ} \mathrm{kg}^{-1}{ }^{\circ} \mathrm{C}^{-1}$ & 2.04 & 2.14 & $2.10 \pm 0.05$ \\
\hline Actividad de agua & $\left(a_{w}\right)$ & 0.607 & 0.612 & $0.604 \pm 0.050$ \\
\hline Humedad & g $100 \mathrm{~g}^{-1}$ & 19.6 & 20.9 & $20.1 \pm 0.43$ \\
\hline $\mathrm{pH}$ & Unidades & 3.82 & 3.93 & $3.87 \pm 0.04$ \\
\hline Acidez Libre & meq $\mathrm{kg}^{-1}$ & 27.9 & 32.0 & $29.7 \pm 1.30$ \\
\hline Acidez Lactónica & meq kg-1 & 1.90 & 2.40 & $2.20 \pm 0.15$ \\
\hline Acidez Total & meq kg-1 & 29.8 & 34.2 & $31.8 \pm 1.40$ \\
\hline Viscosidad & (Pa.s, $\left.25^{\circ} \mathrm{C}\right)$ & 5.12 & 6.10 & $5.61 \pm 0.50$ \\
\hline Tixotropía & $\mathrm{Pa} / \mathrm{s}$ & 10210 & 12610 & $11410 \pm 1671$ \\
\hline Azúcares Reductores & g $100 \mathrm{~g}^{-1}$ & 69.5 & 70.0 & $69.7 \pm 0.17$ \\
\hline Fructosa & g $100 \mathrm{~g}^{-1}$ & 39.7 & 40.4 & $40.1 \pm 0.30$ \\
\hline Glucosa & g $100 \mathrm{~g}^{-1}$ & 29.3 & 30.1 & $29.6 \pm 0.34$ \\
\hline Fructosa/Glucosa & - & 1.32 & 1.38 & $1.35 \pm 0.03$ \\
\hline Fenoles & $\mathrm{mg} \mathrm{kg}^{-1} \mathrm{~A} \cdot \mathrm{Ga}$ & 1582 & 1554 & $1570 \pm 14$ \\
\hline Flavonoides & $\mathrm{mg} \mathrm{kg}^{-1} \mathrm{Fl} . \mathrm{Q}$. & 128.2 & 131.0 & $129.4 \pm 1.20$ \\
\hline Prolina & $\mathrm{mg} \mathrm{kg}^{-1}$ & 317 & 412 & $368 \pm 37.2$ \\
\hline Hidroximetilfurfural (HMF) & $\mathrm{mg} \mathrm{kg}^{-1}$ & 42.9 & 44.7 & $43.8 \pm 0.67$ \\
\hline Índice de Diastasa & Shade & 11.5 & 12.1 & $11.8 \pm 0.30$ \\
\hline \multirow[t]{2}{*}{ Densidad Óptica } & $420 \mathrm{~nm}$ & 0.572 & 0.622 & $0.597 \pm 0.025$ \\
\hline & $635 \mathrm{~nm}$ & 0.102 & 0.132 & $0.117 \pm 0.015$ \\
\hline Índice de Tabouret & - & 3.71 & 3.80 & $3.75 \pm 0.06$ \\
\hline Color Pfund & $\mathrm{mm}$ & 68.0 & 70.0 & $69.0 \pm 1.00$ \\
\hline Luminancia & $\mathrm{L}$ & 58.1 & 61.0 & $59.6 \pm 1.48$ \\
\hline Cromaticidad rojo/verde & $a^{*}$ & 16.5 & 18.4 & $17.4 \pm 0.94$ \\
\hline Cromaticidad amarillo/azul & $b^{*}$ & 77.9 & 81.8 & $79.8 \pm 1.90$ \\
\hline Croma & $\mathrm{C}$ & 79.6 & 83.8 & $81.7 \pm 2.10$ \\
\hline
\end{tabular}

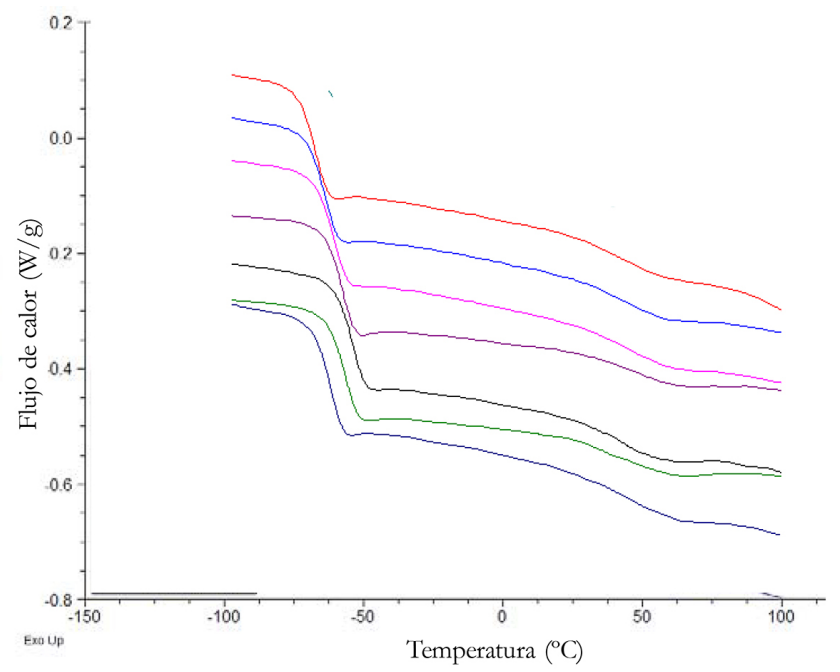

Figura 3. Perfil calorimétrico diferencial de barrido de mieles de Encenillo

condiciones térmicas diferentes, posibilitando la construcción de curvas maestras, cuyos tiempos sobrepasan en gran medida la capacidad de un experimento. ${ }^{69,72,76}$ Las constantes $\mathrm{C} 1$ y $\mathrm{C} 2$ de la expresión WLF en las muestras de Encenillo fueron 2.42 y 40.3, a $10{ }^{\circ} \mathrm{C}$ respectivamente (Figura 5). Estos valores se relacionan por primera vez como de referencia de mieles monoflorales de la zona altoandina colombiana.

\section{Componentes volátiles}

En las mieles de Encenillo se han identificado 24 componentes volátiles (CV). En el análisis de los espectros de masas de los constituyentes individuales, se revela la presencia de compuestos de bajo peso molecular, que representan fragmentos de alcoholes, derivados de ésteres, aldehídos y furfural, que coinciden con reportes de otros estudios. ${ }^{1,36}$ Entre éstos componentes se han identificado 7 alcoholes, 4 aldehídos, 4 cetonas, 1 éster, 3 hidrocarburos, 2 furanos, 1 nitrilos, 1 derivado sulfurado y 2 terpenos. Los alcoholes provienen de la acción fermentativa de las levaduras nativas, los ácidos carboxílicos son formados por efectos de oxidación de alcoholes y aldehídos y sus ésteres por condensación de la fracción ácida con los alcoholes respectivos, la presencia de aldehídos, cetonas y compuestos $\mathrm{C}_{6}$, están relacionados con el metabolismo celular, aspectos ya considerados en la literatura. ${ }^{77} \mathrm{El}$ tolueno, ha sido identificado en 

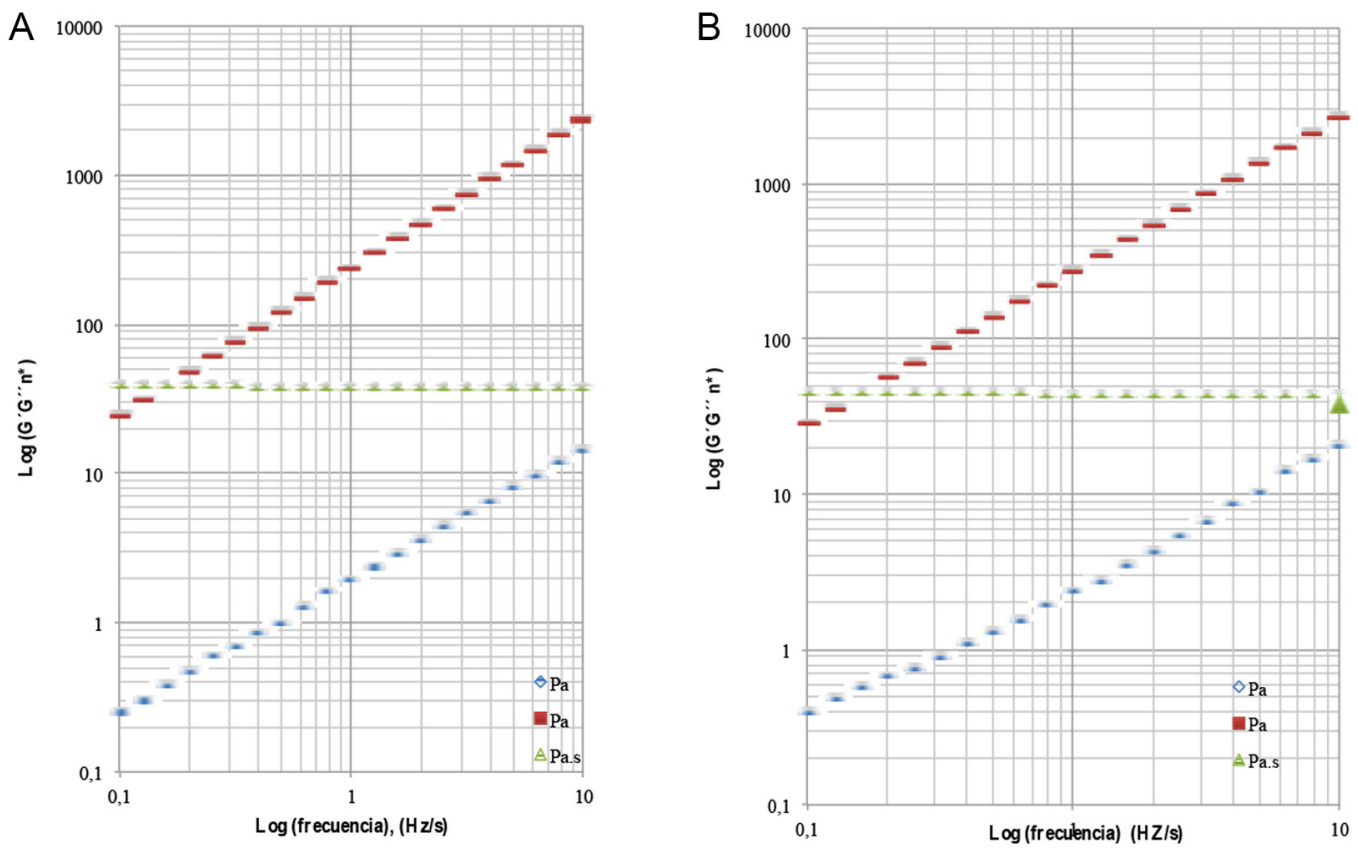

Figura 4. Comportamiento oscilatorio tipo de los módulos de almacenamiento ( $G$ ') pérdida de la viscosidad ( $G$ ”) viscosidad compleja ( $\left.{ }^{*}\right)$ en mieles de Encenillo a 5 (A) y $10^{\circ} \mathrm{C}(B)$

Tabla 2. Valores medios de los módulos de almacenamiento y pérdida de la viscosidad en mieles de Encenillo en función de la temperatura

\begin{tabular}{lcccc}
\hline \multirow{2}{*}{ Temperatura $/{ }^{\circ} \mathrm{C}$} & \multicolumn{3}{c}{$\mathrm{G}^{\prime}$} & \multicolumn{2}{c}{$\mathrm{G}^{\prime \prime}$} \\
\cline { 2 - 5 } & $K^{\prime}(\mathrm{ds})$ & $n^{\prime}(\mathrm{ds})$ & $K^{\prime \prime}(\mathrm{ds})$ & $n^{\prime \prime}(\mathrm{ds})$ \\
\hline 5 & $4.48(0.08)$ & $0.93(0.02)$ & $460(4.88)$ & $0.993(0.003)$ \\
10 & $2.23(0.23)$ & $0.87(0.05)$ & $228(9.50)$ & $0.991(0.002)$ \\
15 & $1.11(0.06)$ & $0.90(0.05)$ & $122(2.20)$ & $0,988(0.007)$ \\
\hline
\end{tabular}

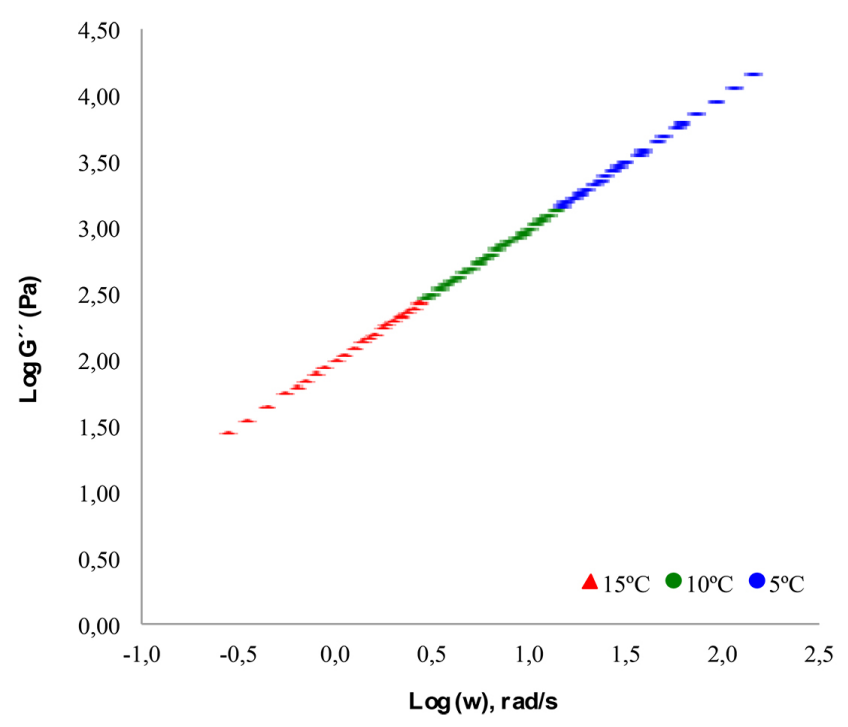

Figura 5. Curva maestra tipo de la relación módulo de pérdida de la viscosidad $(G ")$ como función de la frecuencia $(\omega)$ en mieles de Encenillo a 5 , 10 y $15{ }^{\circ} \mathrm{C}$

mieles y la presencia de éste compuesto se ha relacionado con constituyentes y exudados de plantas. ${ }^{54}$ Los derivados del furano, suelen dependiendo del origen botánico y el tratamiento previo de las mieles producirse por efecto térmico o por degradación enzimática.
En mieles multiflorales y de cítricos, se ha reportado la presencia de benzaldehido, dimetil sulfuro y derivados de furfural. ${ }^{21,78} \mathrm{En}$ mieles florales ${ }^{4,17,33,34,38}$ y monoflorales y algunos de los compuestos identificados en éste trabajo han sido relacionados como responsables directos del aroma. ${ }^{55,57,79-83}$

La mayoría de las mieles indistintamente de su origen botánico, presentan más de un componente de los cuales derivan las propiedades y atributos sensoriales. ${ }^{21}$ La presencia de Acetato de etilo, benzaldehído, bencenacetaldehido, dimetilsulfuro, furfural, limoneno, óxido de linalool y tolueno, se consolidan como marcadores moleculares de la miel de Encenillo y de los cuales derivan las tonalidades de aroma dulce, fragancias florales, ligero sabor y acento resinoso, cuyos valores umbrales de olor no son superiores a 5 partes por billón. La huella digital "Fingerprint" de las mieles de Encenillo se reveló a partir de los fragmentos observados en los espectros de masas de los CV aislados cuyas relaciones características $(\mathrm{m} / \mathrm{z})$ aparecen a 44.6 como más abundante y en proporción media a 38.6 a 40.7; 42.6; 46.6; 54.6 a 61.6 y 72, (Figura 6) identificados y reportados por primera vez para una miel monofloral colombiana. Algunos de éstos fragmentos son coincidentes con los observados en componentes volátiles de otros tipos de miel. 1,17,31,32,57,80 $^{-1}$

\section{CONCLUSIONES}

En las condiciones del estudio y respecto de las valoraciones melisopalinológicas de las mieles, se ha encontrado alta representación del polen de la especie Weinmannia tomentosa Don, que es dominante en los bosques altoandinos colombianos y en particular en el sector de Sotaquirá. El néctar madurado en los panales se expresa como miel de la tonalidad ámbar claro, que además se relaciona con los parámetros de luminancia, cromaticidad rojo/verde y amarillo/ azul, que no habían sido descritos en éstas mieles, que presentan además acento de aroma floral, con leves notas fenólicas y retrogusto resinoso, que marca diferencias importantes con otros tipos de mieles tropicales. Los parámetros fisicoquímicos indicadores de calidad de las muestras no son diferentes de otros tipos de mieles florales y monoflorales descritas en la literatura, la actividad de 
Tabla 3. Principales componentes de la fracción volátil de las mieles de Encenillo $\left(\mathrm{g} \mathrm{kg}^{-1}\right.$ miel) de la zona altoandina de Boyacá

\begin{tabular}{|c|c|c|c|c|c|c|}
\hline Compuesto & Pico & Compuesto & TR & $\mathrm{IK}$ & $\mu \mathrm{g} \mathrm{kg}^{-1}(\mathrm{ds})$ & Identidad \\
\hline \multirow{8}{*}{ Alcoholes } & 12 & Etanol & $5.04-6.62$ & $878-926$ & $185.3(1.91)$ & St; MS; KI \\
\hline & 19 & 1-Butanol & $7.90-9.40$ & 990-1020 & $201(0.40)$ & MS; KI \\
\hline & 15 & 2-Butanol & $7.84-9.36$ & $990-1020$ & $450(0.73)$ & St; MS; KI \\
\hline & 18 & 2-Metil-1-propanol & $10.3-11.9$ & $1067-1120$ & $17.1(1.90)$ & St; MS; KI \\
\hline & 10 & 2-Metil-2-propanol & $3.33-5.59$ & $1124-1240$ & $12.3(0.23)$ & MS; KI \\
\hline & 21 & 2-Metil-1-butanol & $13.4-15.4$ & $1175-1268$ & $22.9(4.03)$ & MS; KI \\
\hline & & 3-Metil-2-butanol & ד. & $1178-1200$ & & \\
\hline & 11 & 2-Propanol & $4.76-6.40$ & $862-916$ & $5.10(0.24)$ & St; MS; KI \\
\hline \multirow{4}{*}{ Aldehídos } & 2 & Acetaldehído & $5.90-5.94$ & $715-761$ & $450(30.1)$ & MS; KI \\
\hline & 5 & 2-Metil propanal & $1.50-2.20$ & $752-794$ & $0.47(0.10)$ & MS; KI \\
\hline & 25 & Benzaldehído & $20.8-22.7$ & $1450-1516$ & $12.1(0.23)$ & MS; KI \\
\hline & 26 & Bencenacetaldehido & $24.5-25.7$ & $1570-1630$ & $0.80(0.05)$ & MS; KI \\
\hline \multirow{4}{*}{ Cetonas } & 9 & 2-Butanona & $4.31-5.72$ & $836-891$ & $20.5(0.40)$ & MS; KI \\
\hline & 6 & 2-Propanon & $1.72-2.41$ & $755-797$ & $38.1(10.6)$ & MS; KI \\
\hline & 22 & 3-Hidroxi-2-butanona & $14.1-15.8$ & $1244-1271$ & $15.8(0.77)$ & St; MS; KI \\
\hline & 13 & 2-Pentanona & $6.41-7.92$ & $937-973$ & $3.20(0,50)$ & MS;KI \\
\hline \multirow{3}{*}{ Hidrocarburos } & 1 & n-Heptano & $1.23-2.42$ & $736-757$ & $0.91(0.06)$ & $\mathrm{St} ; \mathrm{MS} ; \mathrm{KI}$ \\
\hline & 4 & n-Octano & $1.38-3.36$ & $737-757$ & $0.46(0.08)$ & St; MS; KI \\
\hline & 17 & Tolueno & $8.65-10.0$ & $1017-1042$ & $7.40(1.16)$ & MS; KI \\
\hline Esteres & 7 & Acetato de etilo & $3.53-5.29$ & $796-868$ & $3.35(0.06)$ & MS; KI \\
\hline \multirow{2}{*}{ Furanos } & 4 & Furfural & $20.2-22.0$ & $1379-1436$ & $67.3(1.28)$ & $\mathrm{St} ; \mathrm{MS} ; \mathrm{KI}$ \\
\hline & 8 & 2-Metilfurano & $3.72-5.49$ & 802-8979 & $86.7(1.65)$ & St; MS; KI \\
\hline Nitrilos & 14 & 2-Metil-propanonitrilo & $7.35-8.30$ & 976-1002 & $0.08(0.02)$ & MS; KI \\
\hline$\underline{\text { Sulfuros }}$ & 3 & Dimetil disulfuro & $0.80-1.30$ & $727-772$ & $3.75(1.60)$ & MS; KI \\
\hline \multirow{2}{*}{ Terpenos } & 20 & D-Limoneno & $12.3-13.9$ & $1156-1201$ & $4.87(0.35)$ & MS; KI \\
\hline & 23 & Óxido de Linalool & $16.8-18.5$ & $1497-1563$ & $0.57(0.07)$ & MS; KI \\
\hline
\end{tabular}

KI: Indice de Kovats. MS: Espectrometría de masas. St: Estándar. Columna capilar (DB-WAX. SGE. 60 m x 0.32 mm d.i., $1.0 \mu \mathrm{m}$ ),

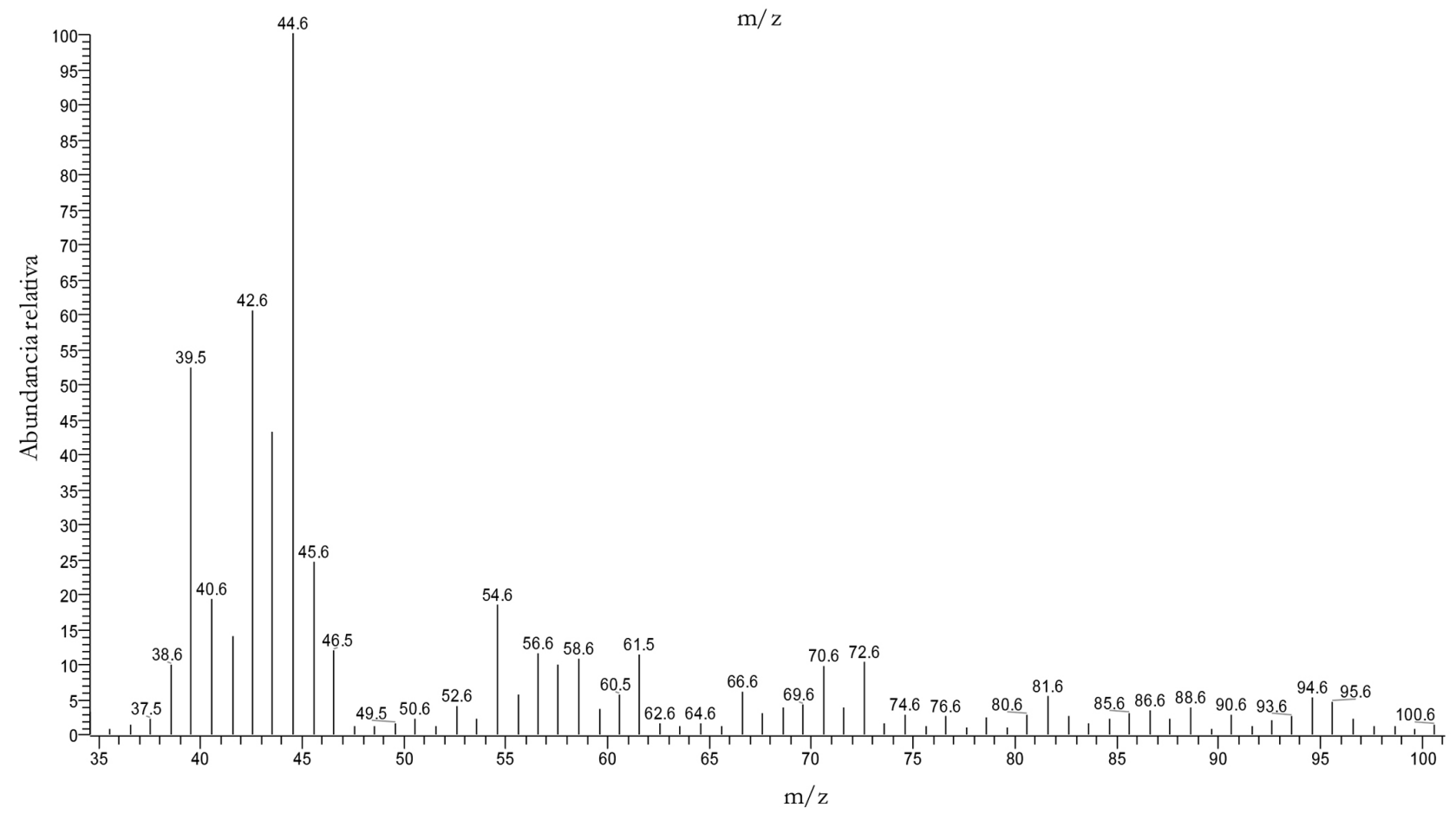

Figura 6. Espectro de masas de los componentes volátiles separados de mieles de Encenillo mediante técnicas de trampa/purga, adsorción/desorción

agua es propia de alimentos de humedad intermedia, el contenido de cenizas, la fracción de cenizas y los sólidos iónicos conductores están correlacionados, dando cuenta de sus propiedades intrínsecas.
Los parámetros fisicoquímicos dan cuenta del grado de madurez alcanzado por el producto tras el proceso de operculación. Las mieles cosechadas expresan su carácter de líquidas con una marcada 
tendencia a la granulación, debido principalmente a la presencia de polen que facilitan su nucleación a la relación de azucares reductores y el contenido de humedad, el carácter es de tenor ácido. El poder rotatorio obedece a las proporciones de fructosa de las cuales deriva el carácter levorotatorio. Se presenta una marcada diferencia en los valores asociados a la densidad óptica a dos longitudes de onda, que se han expresados como valores de absorbancia y que son mayores a bajas longitudes de onda. Los perfiles termográficos, indican la presencia de dos endotermos y la convergencia de las muestras a la transición vítrea. Los valores observados para el contenido de fenoles y flavonoides totales son coincidentes con los atributos sensoriales y dan cuenta de su potencial actividad antioxidante. Las propiedades mecánicas, son propias de un fluido newtoniano, la viscosidad se reduce con la temperatura, los parámetros asociados a los módulos de almacenamiento y pérdida de la viscosidad se incrementan con la frecuencia del proceso oscilatorio. La generación de curvas maestras tras la implementación del modelo de WilliamsLandel-Ferry permitió estimar los parámetros mecánicos de la miel a diferentes frecuencias oscilatorias. En la determinación de componentes volátiles las operaciones de separación purga y trampa, con posterior desorción, permitió la cuantificación de componentes volátiles y el patrón de huella digital de los componentes volátiles. El trabajo relacionado planteó un modelo de investigación y de relación de parámetros que contribuyó al estudio y diferenciación de las mieles colombianas.

\section{AGRADECIMIENTOS}

Los autores expresan su gratitud al Departamento Administrativo de Ciencia Tecnología e Innovación (COLCIENCIAS) y a su programa Jóvenes Investigadores e innovadores 2012. Al grupo de apicultores de la zona altoandina de Boyacá, por haber facilitado las condiciones de muestreo de las mieles, en particular a los señores Efraín Muñoz Casteblanco y Edgar Fernando Vargas Gonzáles. A los profesores Juan Antonio Serra Belenguer e Isabel Escriche Roberto del Instituto de Ingeniería de Alimentos para el Desarrollo (IIAD) de la Universidad Politécnica de Valencia, por el acompañamiento en la gestión y valoraciones analíticas A la señora Roice Eliana Rosim y los profesores Carlos Augusto Oliveira, Izabel Christina Freitas Moraes y Paulo José Amaral do Sobral y de la Faculdade de Zootecnia e Engenharia de Alimentos de la Universidad de São Paulo (Pirassununga), por facilitar parte del equipamento para la evaluación de los azucares, las propiedades térmicas y propiedades reológicas. A los evaluadores del artículo y colaboradores permanentes de la revista Quimica Nova, por las observaciones en el proceso de arbitraje. Los resultados de éste trabajo hacen parte del proyecto 240111, financiado por la Oficina de Investigaciones y Desarrollo Científico de la Universidad del Tolima.

\section{REFERENCIAS}

1. Kus, P. M.; Van Ruth, S.; LWT -- Food Sci. Technol. 2015, 62, 69

2. Tuberoso, C.; Jerkovic, I.; Sarais, G.; Congiu, F.; Marijanovic, Z.; Kus, P.; Food Chem. 2014, 145, 284.

3. Špánik, I.; Pazitná, A.; Šiška, P.; Szolcsányi, P.; Food Chem. 2014, 158, 497.

4. Mayi-Loh, C. E.; Ndip, R. N.; Clarke, A. M.; Int. J. Mol. Sci. 2011, 12, 9514.

5. Soria, A.C.; Gonzalez, M.; de Lorenzo, C.; Castro-Martínez, I.; Sanz, J.; Food Chem. 2004, 85, 121.

6. Wang, J.; Li, Q.; En Chemical Composition, Characterization, and Differentiation of Honey Botanical and Geographical Origin; Taylor, S. L., eds.; Adv. Food Nutr. Res. 2011, cap. 3.
7. Heil, M.; Trends Plant Sci. 2011, 16, 191.

8. González-Teuber, M.; Eilmus, S.; Muck, A.; Svatos, A.; Heil, M.; Plant J. 2009, 58, 464.

9. Moniruzzaman, M.; Amrah Sulaiman, S.; Khalil, M. I.; Hua Gan, S.; Chem. Cent. J. 2013, 7, 1 .

10. Salamanca, G. G.; Rosim, E. M.; Oliveira, C. A.; Sobral, P. J.; Resúmenes VII Congreso Colombiano de Cromatografía, Cartagena de Indias, Colombia, 2014.

11. Persano Oddo, L.; Bogdanov, S.; Apidologie 2004, 35, S2.

12. Bueno-Costa, F.; Zambiazi, C.; Wendt, B.; Clasen, F.; Padilha, W.; Teixeira, J.; Dutra, I.; LWT -- Food Sci. Technol. 2016, 65, 333.

13. Bogdanov, S.; Lüllman, C.; Martin, P.; Von der Ohed, W.; Russmann, H.; Persano Oddo, G.; Sabatini, A.; Marcazzan, G.; Pirof, R.; Flamini, C.; Morlot, M.; Lhéritier, J.; Borneckg, R.; Marioleas, P.; Tsigourih, A.; Kerkvlieti, J.; Ortizj, A.; Ivanovk, T.; D’Arcy, B.; Mosell, B.; Vitm, P.; Harmonised Methods of the International Honey Commission International Honey Commission; Bee world 1999, 80, 61.

14. Jantakee, K.; Traggolpua, Y.; Biol. Res. 2015, 48, 1.

15. Wanjari, N.; Waghware, J.; Int. J. Pharma Res. Rev. 2015, 4, 45.

16. Burlando, B.; Cornara, L.; J. Cosmet. Dermatol. 2013, 12, 306.

17. Panseri, S.; Manzo, A.; Chiesa, L. M.; Giorgi, A.; J. Chem. 2013, 11, 904202.

18. Castro-Várquez L.; Díaz-Maroto M.C.; González-Viñas M.A.; PérezCoello M.S.; Food Chem. 2009, 112, 1022.

19. Gašić, U.; Natić, M.; Mišić, D.; Lušić, D.; Milojković, D.; Tešić, Z.; Lušić, D.; J. Food Compos. Anal. 2015, 44, 128.

20. Jasicka-Misiak, I.; Kafarski, P.; Chemik 2014, 68, 335.

21. Kaškoniené, V.; Maruška, A.; Kornyšova, O.; Chem. Technol. (Kaunas, Lith.) 2009, 52, 74.

22. Karabagias, I.; Badeka, A.; Kontakos, S.; Karabournioti, S.; Kontominas, M.; Food Chem. 2014, 146, 548.

23. Lušić, D.; Koprivnjak, O.; Ćurić, D.; Sabatini, A.; Conte, L.; Food Technol. Biotechnol. 2007, 45(2), 156.

24. Salamanca, G. G.; Moraes, F. I. C.; Bittante, Q. A. M.; Souki, N. G. N.; Sobral P. J. A.; Resumenes del $16^{\text {th }}$ World Congress of Food Science and Technology, Foz do Iguaçu, Paraná, Brasil, 2012.

25. Persano-Oddo L.; Piro R.; Apidologie 2004, 35, S-38

26. Osés, S.; Pascual-Maté, A.; de la Fuente, D.; de Pablo, A.; FernándezMuiño, M.; Sancho, T.; NJAS - Wageningen Journal of Life Sciences 2016, In press.

27. Kropf, U.; Jammik, M.; Bertoncelj, J.; Golob, T.; Food Technol. Biotechnol. 2008, 46, 335.

28. Reyes, M. L. M.; Salamanca, G. G.; Rev. Fac. Nac. Agron., Univ. Antioquia 2014, 67, 461.

29. Talha, K. A.; Khan, I.; Ahmad, K.; Ali, K. Y.; Khan, J.; Khan, Z.; J. Tradit. Chin. Med. 2014, 34(1), 86.

30. Barra, M. P. G.; Ponce-Díaz, M. C.; Venegas-Gallegos, C.; Chil. J. Agr. Res. 2010, 70, 75 .

31. Montenegro, G.; Gómez, M.; Casaubon, G.; Belancic, A.; Mujica, A. M.; Peña, R. C.; Int. J. Exp. Bot. 2009, 78, 61.

32. Jerković I.; Marijanović Z.; Chem. Biodiversity 2009, 6, 421.

33. Gašić, U.; Kečkeš, S.; Dabić, D.; Trifković, J.; Milojković- Opsenica, D.; Natić, M.; Tešić, Ž.; Food Chem. 2014, 145, 599.

34. Cavvaza, A.; Corradini, C.; Musci, M.; Salvadeo, P.; J. Sci Food Agric. 2013, 93, 1169.

35. Kečkeš, S.; Gašić, U.; Veličković, T. Ć.; Milojković-Opsenica, D.; Natić, M.; Tešić, Ž.; Food Chem. 2013, 138, 32.

36. Schuhfried, E.; Sánchez del Pulgar, J.; Bobba, M.; Piro, R.; Cappellin, L.; Märk, T. D.; Franco Biasioli, F.; Talanta 2016, 147, 213.

37. Plutowska B., Chmiel T., Dymerski T., Wardencki W.; Food Chem. 2011, 126,1288

38. Alissandrakis E.; Tarantilis P. A.; Harizanis P. C.; Polissiou M.; J. Sci. Food Agric. 2005, 85, 91. 
39. Moussa, A.; Noureddine, D.; Saad, A.; Abdelmelek, M.; Adbelkader, B.; Asian Pac. J. Trop. Biomed. 2012, 2, 554.

40. Mavric, E.; Wittman, S.; Barth, G.; Henle, T.; Mol. Nutr. Food Res. 2008, 52,483

41. Montoya-López, J.; García-Merchán, V. H.; Lucas-Aguirre, J. C.; Rev. Fac. Nac. Agron., Univ. Antioquia 2014, 67, 51.

42. Velásquez-Giraldo, A. M.; Vélez-Acosta, L. M.; Zuluaga-Gallego, R.; Ing. Cienc. (Medellin, Colomb.) 2013, 9, 61.

43. Chamorro-García, F.; León-Bonilla, D.; Nates-Parra, G.; Colombia Forestal 2013, 16, 53.

44. Gamboa-Abril, M. V.; Figueroa, R. J.; Acta Biol. Colomb. 2014, 14, 97.

45. Salamanca, G. G.; Tesis doctoral, Universidad Politécnica de Valencia, España, 2001

46. Pulido, C. R. M.; Revista de Investigación Agraria y Ambiental 2011, 2 , 45. DOI: $10.22490 / 21456453.910$

47. Montenegro, A.; Vargas, O.; Rev. Biol. Trop. 2008, 56, 705.

48. Luz, C.F.P.; Barth, O.M.; Braz. J. Bot. 2012, 35, 79.

49. Socha, R.; Juszczak, L.; Pietrzyk, S.; Fortuna, T.; Food Chem. 2009, 113, 568.

50. Ferreira, I. C. F. R.; Aires, E.; Barreira, J. C. M.; Estevinho, L. M.; Food Chem. 2009, 114, 1438.

51. Salamanca, G. G.; Osorio, T. M.; Scriche, R. I.; Resúmenes del VII Simposio Internacional de Innovación y Desarrollo de Alimentos, INNOVA, X Congreso Iberoamericano de Ingeniería de alimentos CIBIA, Montevideo, Uruguay, 2015.

52. Rosalina, I.; Bhattacharya, M.; Carbohydr. Polym. 2002, 48, 191.

53. Escriche, R. I.; Visquert, M.; Juan-Borras, M.; Fito P.; Food Chem. 2009, $112,329$.

54. http://www.sisweb.com/referenc/applnote/app-25.htm, accesada en Mayo 2017.

55. De la Fuente, E.; Martínez-Castro, I.; Sanz, J.; J. Sep. Sci. 2005, 28, 1093.

56. Lazaridou, A.; Biliaderis, C. G.; Bacandritsos, N.; Sabatini, A.; J. Food Eng. 2004, 64, 9

57. Kadar, M.; Juan-Borrás, M.; Carot, J. M.; Domenech, E.; Escriche, I.; J. Sci. Food Agric. 2011, 91(15), 2768.

58. Akbulut, M.; Özcan, M.M.; Çoklar, H.; Int. J. Food Sci. Nutr. 2009, 60, 577.

59. De la Fuente. M.L.; Sanz, J.; Martínez-Castro, I.; Ruiz-Matute, A. I.; Food Chem. 2007, 105, 84.

60. Aljadi, A. M.; Kamaruddin, M. Y.; Food Chem. 2004, 85, 513.

61. Liberato, M.; Morais, S. M.; Siqueira, S.; Menezes, J, E.; Ramos, D. N.; Machado, L.; Magalhães, I. L.; J. Med. Food 2011, 14, 658.
62. Qamer, S.; Ehsan, M.; Nadeem, S.; Abdul Rauf Shakoori, A. R.; Pak. J. Zool. 2007, 39, 99.

63. Alqarni, A.; Owayss, A.; Mahmoud, A.; Arabian J. Chem. 2016, 9, 114.

64. Batista, J.; Leite, E.; Marques, G.; de Toledo, M.; Gullón, B.; Pintado, M.; Magnani, M.; LWT -- Food Sci. Technol. 2016, 65, 645.

65. Bentabol, A.; Hernández, Z.; Rodríguez, B.; Rodríguez, E.; Díaz, C.; LWT -- Food Sci. Technol. 2014, 55, 572

66. Dinkov, D.; Apidologie 2003, 34, 319.

67. Přidal, A.; Vorlová, L.; Czech J. Anim. Sci. 2002, 47, 439.

68. Persano-Oddo, L.; Piazza, M. G; Sabatini, A. G.; Apidologie 1995, 26 , 453.

69. Bakier, S.; Pol. J. Food Nutr. Sci. 2007, 57 (2A), 7.

70. Cavia M. M.; Fernández-Muíno, M. A.; Huidobro J. F.; Álvarez, C.; Sancho, M. T.; Food Chem. 2007, 100, 1728.

71. Cordella, C.; Moussa, I.; Martel, A.; Sbirrazzuoli, N.; Lizzani-Cuvelier, L.; J. Agric. Food Chem. 2002, 50, 1751.

72. Sopade, P. A.; Halley, P.; Bhandari, B.; D’Arcy, B.; Doebler, C.; Caffin, N.; J. Food Eng. 2003, 56, 67.

73. Ahmed, J.; Prabhu, S.; Raghavan, G.; Ngadi, M.; J. Food Eng. 2007, 79, 1207.

74. Costa, P. A.; Moraes, I. C. F.; Bittante, A. M. Q. B.; Sobral, P. J. A.; Gomide, C. A.; Carrer, Da Costa, C.; Abstracts of Proceedings of ICEF11, Atenas, Grecia, 2011.

75. Chen, Y. W.; Lin, C. H.; Wu, F. Y.; Chen, H. H.; J. Food Process Eng. 2009, 32, 512 .

76. Gasparoux, J.; Laux, D.; Ferrandis, J. Y.; Attal, J.; Tordjeman, P.; J. NonNewtonian Fluid Mech. 2008, 153, 46.

77. Solinas, M.; Angerosa, F.; Cucurachi, A.; Rivista della Società Italiana di Scienze dell'Alimentazione 1985, 14, 361.

78. Wardencki, W.; Chmiel, T.; Dymerski, T.; Biernacka, P.; Plutowska, B.; Ecol. Chem. Eng. 2009, 16, 287.

79. Cuevas-Glory, L. F.; Pino, J. A.; Santiago, L. S.; Sauri-Duch, L. F.; Food Chem. 2007, 103, 1032.

80. Castro-Várquez L. M.; Díaz-Maroto M. C.; Pérez-Coello M. S.; J. Agric. Food Chem. 2006, 54, 4809

81. Baroni, M. V.; Nores, M. L.; Díaz, M. D. P.; Chiabrando, G. A.; Fassano, J. P.; Costa, C.; Wunderlin, D. A.; J. Agric. Food Chem. 2006, 54, 7235.

82. Wolski, T.; Tambor, K.; Rybak-Chmielewska, H.; Kêdzia, B.; J. Api. Sci. 2006, 50, 115.

83. Bastos, C.; Alves, R.; Quim. Nova 2003, 26, 90 